\title{
Análisis de la pigmentación cutánea en una muestra
}

\author{
Juan Pablo Castanedo-Cazares', Diana Hernández-Blanco', Juan Diego García-Cortés², \\ Liliana Medina-Aguilar ${ }^{1}$ y Bertha Torres-Álvarez ${ }^{1}$ \\ ${ }^{1}$ Departamento de Dermatología, Hospital Central Dr. Ignacio Morones Prieto; ${ }^{2}$ Departamento de Inmunología y Biología Molecular, Facultad de \\ Ciencias Químicas. Universidad Autónoma de San Luis Potosí, San Luis Potosí. S.L.P., México
}

\section{Resumen}

Antecedentes: La mezcla de melanina, hemoglobina y carotenos definen el color cutáneo. La pigmentación constitutiva está determinada genéticamente, la facultativa se induce cuando la piel se expone al ambiente. El objetivo fue cuantificar ambas pigmentaciones en una muestra de población mexicana, y analizar su relación con el género, edad y fototipo. Métodos: Se evaluaron 259 personas durante un periodo invernal. La colorimetría cutánea se obtuvo mediante espectrometría de reflectancia difusa utilizando las coordenadas de la Comisión Internacional de lluminación. Se registraron los valores $L^{*} a^{*} b^{*}$ y se estimó el ángulo tipológico individual $\left(A T I^{\circ}\right)$ en frente, tórax, cuello, antebrazos y glúteos. Resultados: La pigmentación facultativa difirió de la constitutiva en los parámetros $L^{*}, a^{*}$, y ATI․ En hombres, los valores facultativos de $L^{*}$ y $A T I^{\circ}$ fueron menores. La pigmentación constitutiva fue similar entre sexos. Los fototipos III, IV y V muestran diferencias en $L^{*}, b^{*}$ y $A T I^{\circ}$. Los valores facultativos $L^{*}, a^{*}, A T I^{\circ}$ y el constitutivo $a^{*}$ se reducen al incrementarse la edad. Conclusiones: Se cuantificaron los tonos cutáneos de una muestra de población reconociéndose los valores para la piel blanca, morena clara y morena oscura. Se presenta un marco de referencia para estudios relacionados con la pigmentación cutánea en México.

PALABRAS CLAVE: Espectrofotometría. Colorimetría. Pigmentación. Fototipo.

\begin{abstract}
Background: The blend of hemoglobin, carotenes, and melanin defines the skin color. Constitutive pigmentation is genetically determined, facultative color is induced when skin is exposed to environment. The objective was to quantify both pigmentations in a sample of Mexican population and to analyze its relationship with sex, age, and phototype. Methods: We evaluated 259 individuals during the winter. Skin colorimetry was obtained by diffuse reflectance spectrometry, using the International Commission of Illumination coordenates. $L^{*} a^{*} b^{*}$ parameters were measured and the individual typological angle (ITA) was estimated from forehead, thorax, neck, forearms, and buttocks areas. Results: Facultative pigmentation differed from constitutive in $L^{*}, a^{*}$, and ITA ${ }^{\circ}$ values. In men, $L^{*}$ and ITA ${ }^{\circ}$ parameters were lower. Constitutive pigmentation was similar between sexes. Phototypes III, IV, and $V$ showed differences in $L^{*}, b^{*}$, and ITA $A^{\circ}$. Facultative values such as $L^{*}, a^{*}, A T I^{\circ}$, and the constitutive $a^{*}$ reduce as age increases. Conclusions: The cutaneous tones of a sample of population were quantified recognizing their values for white, light brown, and dark brown skin. A reference frame for research related to cutaneous pigmentation in Mexico is presented.
\end{abstract}

KEY WORDS: Spectrophotometry. Colorimetry. Pigmentation. Phototype.

\author{
Correspondencia: \\ Bertha Torres-Álvarez \\ Venustiano Carranza No. 2395 \\ Zona Universitaria \\ C.P. 78210, San Luis Potosí, S.L.P., México \\ E-mail: torresmab@yahoo.com.mx
}

Fecha de recepción en versión modificada: 29-11-2016

Fecha de aceptación: 04-01-2017

DOI://dx.doi.org/10.24875/GMM.17003050
Gac Med Mex. 2018;154:68-73

Contents available at PubMed www.gacetamedicademexico.com 


\section{Introducción}

La piel humana es diversa en su color, ya que exhibe una gama de tonos blancos, morenos y negros. Esta característica se utiliza para distinguir o clasificar las diferentes razas ${ }^{1}$. Su aspecto se debe a la mezcla de pigmentos o cromóforos que absorben determinadas longitudes electromagnéticas y las transmiten 0 reflejan en el espectro de luz visible ${ }^{2-4}$. Los más importantes son la oxihemoglobina, que refleja el color rojo, la desoxihemoglobina, que refleja el azul, los carotenos, que reflejan el amarillo-naranja y la melanina, que refleja el marrón ${ }^{3,5,6}$. La melanina es el principal pigmento que determina el tono oscuro o claro según su concentración en la epidermis ${ }^{2,3}$. La función biológica de esta sustancia es proteger a las células de la radiación ultravioleta (UV) y su síntesis se incrementa en respuesta a estímulos ambientales ${ }^{7.9}$.

El color determinado genéticamente que se observa en zonas del cuerpo aisladas del ambiente y/o protegidas por la vestimenta representa la pigmentación constitutiva ${ }^{10}$. El oscurecimiento ocasionado por la exposición UV corresponde a la pigmentación facultativa. Este bronceamiento es evidente en zonas expuestas como cara, cuello, brazos y antebrazos, e involuciona gradualmente al cesar el estímulo ${ }^{8,10}$. Por lo tanto, la observación de la pigmentación cutánea y sus cambios sugieren la tolerancia o predisposición para el desarrollo de patologías asociadas a la radiación solar9,11. Si la agresión UV excede los mecanismos de protección o ajuste; quemaduras, discromías, envejecimiento y neoplasias benignas o malignas aparecerán sobre las zonas más expuestas de la piel $^{8,12,13}$.

Debido a la percepción subjetiva del color cutáneo, la clasificación de Fitzpatrick se emplea en medicina para reconocer el tipo de piel; esta evalúa su respuesta biológica a la exposición solar distinguiendo seis tonos o fototipos de piel, con tres variantes de piel blanca, dos morenas y la negra ${ }^{14}$. Sin embargo, la cuantificación objetiva del color de la piel requiere la localización tridimensional de los colores rojo, verde y azul ${ }^{3}$. Estas coordenadas se basan en las bandas de absorción de los tres pigmentos presentes en los conos de la retina humana ${ }^{15}$. La colorimetría consiste en la medición objetiva de estos tres valores, así como su luminiscencia, mediante algún método como el propuesto por la Comisión Internacional sobre Iluminación (V.gr. L*a* $\left.a^{*}\right)^{3,15}$. Este proceso permite la representación numérica de cualquier color presente en la naturaleza ${ }^{3,4,15}$. El propósito de este trabajo fue cuantificar la pigmentación constitutiva y facultativa en personas adultas mexicanas utilizando un espectrómetro de reflectancia difusa y analizar su relación con el género, edad y fototipo.

\section{Métodos}

Mediante consentimiento informado y aprobación por el comité de ética local (3611), se invitó a participar a voluntarios mayores de 18 años durante el periodo invernal de noviembre a febrero. Mediante muestreo no probabilístico se incluyeron usuarios y visitantes del Hospital Central Dr. Ignacio Morones Prieto de la ciudad de San Luis Potosí. Se seleccionaron personas con cualquier tono de piel que contaran con la nacionalidad mexicana desde al menos tres generaciones previas. Se excluyeron personas con enfermedades cutáneas y sistémicas, ingesta de fármacos, embarazo o lactancia durante seis meses previos.

El tipo de piel se evaluó según la clasificación de Fitzpatrick ${ }^{14}$, que examina la respuesta cutánea de una persona después una hora de exposición solar a mitad del día en el verano. Este intervalo contiene aproximadamente $90 \mathrm{~mJ} / \mathrm{cm}^{2}$ de radiación UV en la mayor parte de la República Mexicana ${ }^{9}$. Después del contacto, y según la existencia de quemadura y/o pigmentación en la piel a las 24 horas y 7 días respectivamente, las respuestas cutáneas se agrupan en: I, siempre se quema, nunca se oscurece; II, se quema pero oscurece con dificultad; III, se quema con oscurecimiento escaso; IV, se quema ocasionalmente con oscurecimiento evidente; $\mathrm{V}$, quemadura ausente con oscurecimiento profuso y VI, quemadura ausente con oscurecimiento permanente ${ }^{9,14}$.

La colorimetría cutánea se midió con un espectrómetro de reflectancia difusa (Chromameter 310, Minolta, Osaka, Japón) utilizando los espacios de color de la Comisión Internacional sobre lluminación. Consisten en un eje central de luminosidad $L^{*}$ con valores de negro (0) a blanco (100) y dos ejes ortogonales que representan la cromaticidad: $a^{*}$ describe los colores del rojo $(+50)$ al verde $(-50)$ y $b^{*}$ los colores del azul $(-50)$ al amarillo $(+50)^{4}$.

La pigmentación facultativa se estimó considerando los promedios de las mediciones obtenidas en cinco sitios corporales: la región supraglabelar en la frente, la zona infraclavicular de tórax superior, el cuello posterior a nivel de la sexta vértebra cervical y la porción lateromedial de ambos antebrazos sobre el epicóndilo. 
La pigmentación constitutiva se obtuvo del promedio de las mediciones sobre el cuadrante superior externo e inferior interno del área glútea. El instrumento fue calibrado antes de cada una de las mediciones y el valor analizado fue la media de tres lecturas consecutivas sobre cada área anatómica descrita.

Los valores de ambas pigmentaciones fueron clasificados de acuerdo al ATI ${ }^{\circ}$. Este valor corresponde a la segmentación angular de la proyección bidimensional de los valores centrales de luminiscencia $\left(L^{*}=50\right)$, contra el valor inicial de la cromaticidad amarilla $\left(b^{*}\right.$ $=0)^{6}$. Se obtiene mediante la fórmula: $\mathrm{ATI}^{0}=[$ Arc$\left.\operatorname{Tan}\left(L^{*}-50\right) / b^{*}\right] \times(180 / 3.14159)$. Este parámetro permite la clasificación de la piel en seis grupos (muy claro > $55>$ claro $>41>$ intermedio $>28>$ bronceado $>10$ $>$ moreno $>-30>$ oscuro) 6,16 .

Aproximadamente el $95 \%$ de los fototipos de la po-

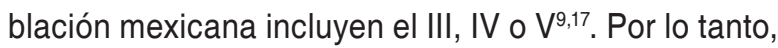
el tamaño de la muestra se calculó en al menos 16 sujetos por grupo según las siguientes consideraciones: una diferencia en la luminosidad $\left(\mathrm{L}^{*}\right)$ del área glútea entre los fototipos II y V de al menos 5\% (V.gr. 61 para tipo III y 58 en el V). La distribución intermedia de las medias, desviación estándar de 1.8 y un intervalo de confianza del $95 \%$, dos colas, alfa de 0.05 y beta de 0.9. El análisis descriptivo se realizó utilizando medidas de tendencia central. La comparación de los datos se realizó mediante prueba t de Student, análisis de varianza y pruebas de correlación. Todo a un nivel de confianza del 95\%, y un nivel de significancia del $5 \%$, utilizando el programa JMP versión 8.0 (Cary, NC, E.U.A.).

\section{Resultados}

Se analizaron las características de 259 personas. La edad promedio fue de $34 \pm 14$ años (rango 18 a $75)$. El $65 \%$ fueron mujeres $(n=169)$, el $35 \%(n=90)$ hombres. Los fototipos de la muestra fueron II $(n=17)$; III $(n=49)$; IV $(n=82)$ y V $(n=111)$. No hubo diferencia en el promedio de edad, ni en las proporciones de género en los cuatro grupos.

El promedio general de los valores de $L^{*}, a^{*}, b^{*} y$ $\mathrm{ATI}^{\circ}$ de la pigmentación facultativa fueron $57.3 \pm 4.7$, $10.2 \pm 2.4,15.8 \pm 2.5$ y $24.2 \pm 14.6$, respectivamente. En relación a la pigmentación constitutiva, los valores medios de $L^{*}, a^{*}, b^{*}$ y $A T I^{\circ}$ fueron $62.7 \pm 4.5,7.7 \pm 2.9$, $14.6 \pm 3$ y $40.3 \pm 15.1$, respectivamente. Todos estos parámetros, a excepción de $b^{*}$, mostraron diferencias significativas entre ambas pigmentaciones $(p<0.01)$.
Tabla 1. Comparación de los valores $L^{*}, a^{*}, b^{*}, y \mathrm{ATI}^{\circ}$ entre hombres y mujeres para las áreas expuestas y no expuestas al ambiente de 259 personas.

\begin{tabular}{lcccccccc}
\hline \multicolumn{1}{l}{ Facultativa } & \multicolumn{6}{c}{ Constitutiva } \\
\hline \multicolumn{1}{c}{ Mujer } & Hombre & $\boldsymbol{P}$ & & Mujer & Hombre & $\boldsymbol{P}$ \\
\hline $\mathrm{L}^{*}$ & $58 \pm 3.9$ & $56.1 \pm 5.7$ & 0.001 & $\mathrm{~L}^{*}$ & $63.1 \pm 4.4$ & $61.8 \pm 5.4$ & 0.06 \\
$\mathrm{a}^{*}$ & $9.7 \pm 2.2$ & $10.2 \pm 2.6$ & 0.08 & $\mathrm{a}^{*}$ & $7.1 \pm 2.7$ & $7.2 \pm 2.9$ & 0.09 \\
$\mathrm{~b}^{*}$ & $15.8 \pm 2.4$ & $15.7 \pm 2.6$ & 0.8 & $\mathrm{~b}^{*}$ & $14.3 \pm 2.8$ & $15 \pm 3.2$ & 0.24 \\
ATIO $^{\circ}$ & $26.5 \pm 12.2$ & $20 \pm 17.5$ & 0.006 & ATI $^{\circ}$ & $41.9 \pm 14$ & $37.4 \pm 16.8$ & 0.08 \\
\hline
\end{tabular}

Los datos muestran la media \pm desviación estándar de $L^{*}$ (luminosidad); $\mathrm{a}^{\star}$ (rojo); b* (azul); ATI (Ángulo Tipológico Individual). Facultativa corresponde a la piel expuesta a radiación solar; constitutiva a la piel no expuesta. $P<0.05$, $t$ de Student.

La comparación entre géneros mostró diferencias significativas de los valores $L^{*}$ y $A T I^{\circ}$ en la pigmentación facultativa, ya que sus valores fueron mayores en los hombres. Sin embargo, la cuantificación de los colores rojos y amarillos fue similar para ambos grupos en ambas pigmentaciones. Estos datos se muestran en la tabla 1.

El cálculo del $\mathrm{ATI}^{\circ}$ para la pigmentación constitutiva estableció que el $15.4 \%(n=40)$ fueron muy claros, el $38.9 \%(n=101)$ claros, el $27.4 \%(n=71)$ intermedios, el $13.2 \%(n=34)$ bronceados, el $5.1 \%(n=13)$ morenos y el $0 \%$ oscuros. El ATIo de la pigmentación facultativa mostró una proporción de $0.7 \%(n=2)$ para piel muy clara, $10.4 \%(n=27)$ para clara, $32 \%(n=83)$ para intermedia, 38.9\% $(n=101)$ para bronceada, $17.7 \%(n=46)$ para morena y $0 \%$ oscura. Esta distribución se muestra en la figura 1.

El análisis de la pigmentación facultativa y constitutiva con respecto a los fototipos de Fitzpatrick demostró diferencias significativas para ambas pigmentaciones en los valores $L^{*}, b^{*}$ y ATIO (ANOVA, $p<0.001$ ). La pigmentación facultativa mostró incremento del valor $L^{*}$ en relación a la constitutiva, de $7.5 \%, 7.5 \%$, $9.2 \%$, y $8.5 \%$ para los fototipos II, III, IV y V, respectivamente. No existieron cambios en el color rojo $\left(\mathrm{a}^{\star}\right)$ entre fototipos. Estos datos se resumen en la tabla 2.

Con respecto a la asociación entre pigmentación y edad, se observó que en la variante facultativa los valores $L^{*}$ y $A T I^{\circ}$ se redujeron conforme aumentó la edad ( $R:-0.17$ y -0.15 respectivamente; $p<0.01$ ). Esta misma tendencia se observó con la representación del tono rojo $\left(a^{*}\right),(R:-0.15 ; p=0.01)$. La pigmentación constitutiva mostró una relación inversa entre la intensidad del color rojo y el incremento de la edad (R: - $0.4 ; p<0.001$ ). No se determinaron cambios en los valores de $L^{*}, b^{*}$ y $A T I^{\circ}$ con relación a la edad. Estos hallazgos se ilustran en la figura 1. 

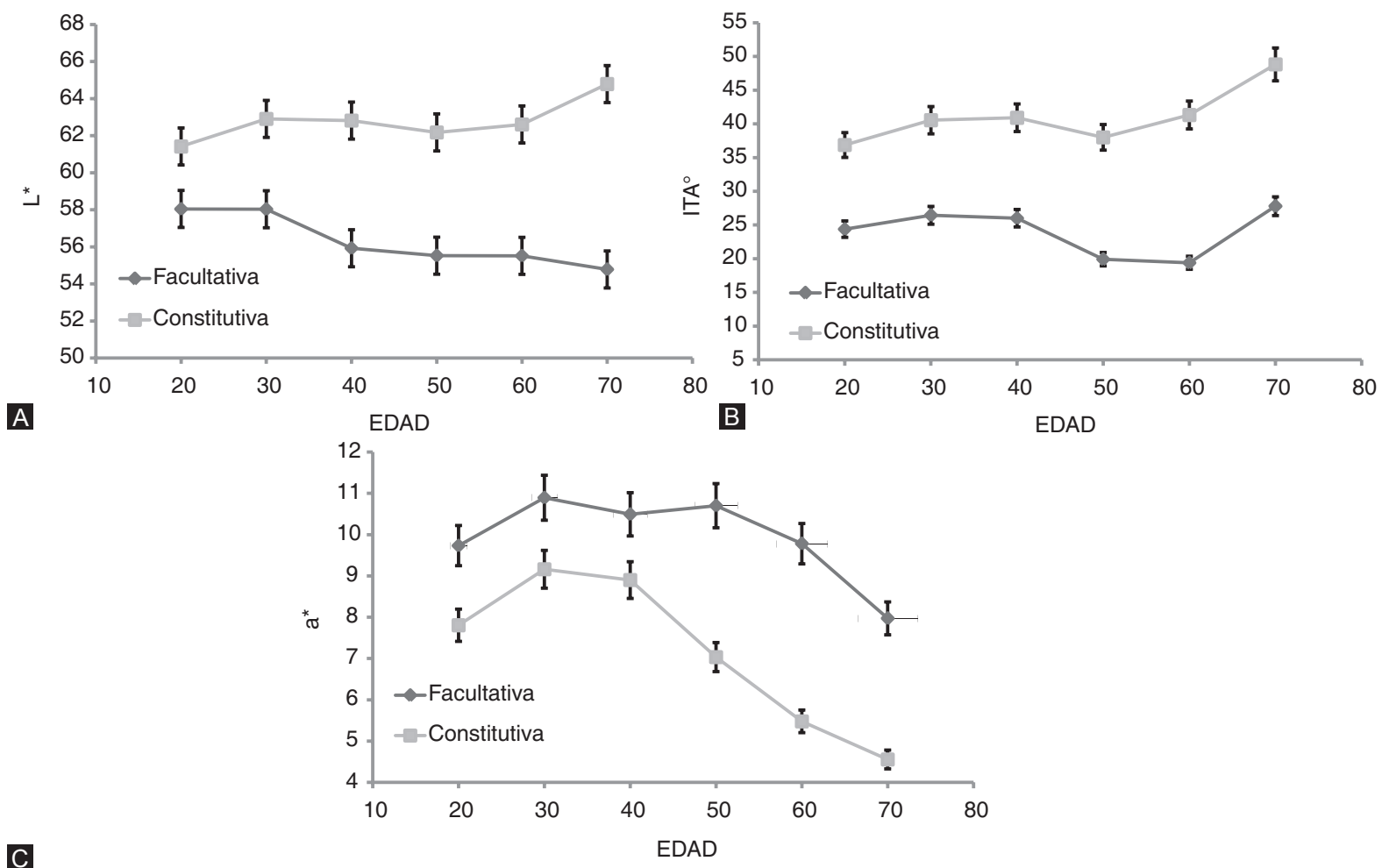

C

Figura 1. Variación de los valores $L^{*}, A T I^{\circ}$ (Ángulo Tipológico Individual), a*en relación a la edad cronológica de los individuos evaluados. (A) La luminosidad se reduce conforme progresa la edad en la pigmentación facultativa $(R-0.17, P<0.01)$, a diferencia de la constitutiva que permanece sin cambio. (B) EI ATI ${ }^{\circ}$ se reduce al incrementarse la edad en la pigmentación constitutiva $(R-0.15, P 0.01)$, y no se modifica en la constitutiva. (C) El valor de $a^{*}$ disminuye al incrementarse la edad en la pigmentación facultativa ( $R-0.15, P$ 0.01), así como en la constitutiva ( $R-0.39, P<0.001)$. La gráfica muestra los valores agrupados por década (media $\pm D E$ ).

Tabla 2. Diferencias entre los valores $L^{*}, a^{*}, b^{*}, y$ ATI ${ }^{\circ}$ de acuerdo a los diferentes fototipos en las áreas expuestas y no expuestas de la piel en 259 personas.

\begin{tabular}{|c|c|c|c|c|c|}
\hline \multicolumn{6}{|c|}{ Facultativa } \\
\hline & II & III & IV & v & $P$ \\
\hline$L^{*}$ & $62.17 \pm 3.12$ & $61.19 \pm 3.87$ & $57.82 \pm 4.14^{\mathrm{a}}$ & $54.66 \pm 3.74^{b}$ & $<0.001$ \\
\hline$a^{*}$ & $10.4 \pm 1.78$ & $9.76 \pm 2.25$ & $9.9 \pm 2.61$ & $10.2 \pm 1.44$ & 0.2 \\
\hline$b^{*}$ & $15.18 \pm 2.53$ & $14.29 \pm 2.5$ & $15.22 \pm 1.99$ & $17.09 \pm 2.28^{b}$ & $<0.001$ \\
\hline$A T I^{\circ}$ & $38.47 \pm 9.85$ & $37.18 \pm 8.04$ & $26.39 \pm 12.71^{\mathrm{a}}$ & $14.78 \pm 11.84^{b}$ & $<0.001$ \\
\hline \multicolumn{6}{|c|}{ Constitutiva } \\
\hline & II & III & IV & v & $P$ \\
\hline$L^{*}$ & $67.25 \pm 3.14$ & $66.17 \pm 3.12$ & $63.79 \pm 3.46^{a}$ & $59.7 \pm 4.7^{b}$ & $<0.001$ \\
\hline$a^{*}$ & $8.49 \pm 2.77$ & $7.13 \pm 2.85$ & $7.18 \pm 2.97$ & $7.85 \pm 2.5$ & 0.06 \\
\hline$b^{*}$ & $13.03 \pm 1.87$ & $12.09 \pm 2.34$ & $13.88 \pm 2.46^{\mathrm{a}}$ & $16.51 \pm 2.61^{b}$ & $<0.001$ \\
\hline$A T I^{\circ}$ & $52.44 \pm 8.15$ & $53 \pm 6.69$ & $44.49 \pm 10.84^{a}$ & $29.88 \pm 14.38$ b & $<0.001$ \\
\hline
\end{tabular}

Los datos muestran la media \pm desviación estándar de $\mathrm{L}^{*}$ (luminosidad); $\mathrm{a}^{*}$ (rojo); b* (azul); ATI (Ángulo Tipológico Individual). Facultativa corresponde a la piel expuesta a radiación solar; constitutiva a la piel no expuesta. $P<0.05$, análisis de varianza (ANOVA); a Diferencia significativa en relación a grupos II, III, V; ${ }^{\circ}$ Diferencia significativa en relación a grupos II, III, IV 


\section{Discusión}

La pigmentación es un rasgo físico que favorece la distinción de los diferentes grupos humanos. Su cuantificación tiene implicaciones antropológicas, sociales, psicológicas y sanitarias ${ }^{10,13,18}$. Esta variable representa un proceso adaptativo al entorno geográfico con repercusiones clínicas. Por lo tanto, medir la pigmentación en una población tiene relevancia ya que traduce la predisposición para sufrir daño ambiental, así como la posible presencia de patologías locales o sistémicas ${ }^{5,16,19}$. Este trabajo representa el primer estudio en México que mide la coloración cutánea mediante reflectometría difusa. El estudio fue realizado durante el invierno, para minimizar la influencia de la intensa exposición solar de los habitantes de nuestro país durante la mayor parte del año ${ }^{17,20}$.

Nuestro estudio encontró un aumento significativo de los valores que indican la presencia de melanina y contenido sanguíneo en la pigmentación facultativa en ambos géneros. Sin embargo, los hombres presentaron valores más elevados que las mujeres. El incremento en la síntesis de melanina en este grupo coincide con informes en relación a un mayor tiempo de exposición solar durante sus actividades laborales y recreativas ${ }^{17}$. En relación a la pigmentación constitutiva, no se hallaron diferencias entre ambos géneros. Esto podría significar que la sensibilidad genética a la radiación UV de nuestra población no se encuentra influenciada de forma determinante por esta variable, como se informa en otros estudios ${ }^{9,15,16,19}$.

En relación a la clasificación clínica de Fitzpatrick, identificamos cuatro de los seis tipos descritos. Aunque la reflectometría no distinguió diferencias entre los valores de fototipos claros como el II y el III, los parámetros $L^{*}$ y $A T I^{\circ}$ identificaron tres tonos o categorías de piel, tanto en la pigmentación facultativa como en la constitutiva. Estos corresponderían al V (moreno oscuro), IV (moreno claro) y a los grupos II-III (blanco). A diferencia de los grupos moreno oscuro y blanco, la divergencia entre ambas pigmentaciones fue más acentuada en el grupo moreno claro. Este mayor contraste podría representar un mayor esfuerzo adaptativo de este grupo en relación a la mejor tolerancia UV de la población morena oscura ${ }^{9,16}$. El escaso contraste entre ambas pigmentaciones en el grupo con piel blanca, demuestra su limitada capacidad para responder a la agresión ambienta|8,10,14.

Otro hallazgo fue el incremento de la pigmentación facultativa conforme progresa la edad. Esto puede relacionarse al incremento de la melanogénesis como mecanismo intrínseco de protección debido al debilitamiento estructural cutáneo y/o a la menor capacidad de depuración melánica propia del envejecimiento cutáneo $0^{8,13,21}$. Sin embargo, la pigmentación constitutiva fue similar entre los distintos grupos etarios analizados. Por otra parte, se observó la reducción del tono rojo conforme se incrementa la edad en ambas pigmentaciones, aunque se encontró más pronunciada en la constitutiva. Este color traduce la presencia de sangre y es probable que lo hallado se deba a la menor vascularidad cutánea que acompaña al envejecimiento cronológico ${ }^{8,22}$.

Identificar los valores de los tres ejes cromáticos que conforman las diversas tonalidades cutáneas es relevante para la medicina de nuestro país, ya que favorece el agrupamiento o tipificación de nuestros habitantes. Esto permite estimar la probabilidad de padecer trastornos de la pigmentación, fotoenvejecimiento, así como tumores benignos o malignos ${ }^{9,13}$. También facilita el establecimiento de medidas preventivas desde etapas tempranas de la vida en la población de piel clara ${ }^{9,12}$. Por otra parte, la identificación de valores cromáticos extremos sugiere la presencia o puede ser útil para monitorear enfermedades que modifican el color tegumentario. Algunos ejemplos son los trastornos de melanogénesis, angiogénesis y el depósito de pigmentos endógenos como bilirrubinas o exógenos como metales pesados ${ }^{5}$.

En conclusión, obtuvimos los rangos cuantitativos de los tonos cutáneos en una muestra de población mexicana utilizando las coordenadas $L^{*} a^{*} b^{*}$ y el $\mathrm{ATI}^{\circ}$. Se observó una mezcla de tonos que se distinguen como piel blanca, morena clara y morena oscura. La pigmentación constitutiva fue similar entre géneros y los distintos grupos de edad. La facultativa se incrementa conforme avanza la edad, siendo más profusa en la población masculina. Estos valores representan un marco de referencia para estudios futuros.

\section{Bibliografía}

1. Relethford JH. Race and global patterns of phenotypic variation. Am J Phys Anthropol. 2009;139:16-22.

2. Anderson RR, Parrish JA. The optics of human skin. J Invest Dermatol. 1981;77:13-9.

3. Takiwaki H. Measurement of skin color: practical application and theoretical considerations. J Med Invest. 1998;44:121-6.

4. Weatherall IL, Coombs BD. Skin color measurements in terms of CIELAB color space values. J Invest Dermatol. 1992;99:468-73.

5. Ortonne JP. Normal and abnormal skin color. Ann Dermatol Venereol. 2012;139 Suppl: S125-9.

6. Chardon A, Cretois I, Hourseau C. Skin colour typology and suntanning pathways. Int J Cosmet Sci. 1991;13:191-208.

7. Nielsen KP, Zhao L, Stamnes JJ, et al. The importance of the depth distribution of melanin in skin for DNA protection and other photobiological processes. J Photochem Photobiol B. 2006;82:194-8. 
8. Hönigsmann $\mathrm{H}$. Erythema and pigmentation. Photodermatol Photoimmunol Photomed. 2002;18:75-81.

9. Castanedo Cázares JP, Torres Álvarez B, Sobrevilla Ondarza S, et al. Estimación del tiempo de exposición solar para quemadura en población mexicana. Gac Med Mex. 2012;148:243-7.

10. Quevedo WC, Fitzpatrick TB, Pathak MA, et al. Role of light in human skin color viariation. Am J Phys Anthropol. 1975;43:393-408.

11. Damian DL, Matthews YJ, Phan TA, et al. An action spectrum for ultraviolet radiation-induced immunosuppression in humans. $\mathrm{Br} \mathrm{J}$ Dermatol. 2011;164:657-9.

12. Castanedo-Cázares JP, Martínez-Rosales K, Hernández-Blanco D, et al. In vitro assessment of commercial sunscreens available in Latin America. Invest Clin. 2014;55:142-54.

13. Diffey BL. Ultraviolet radiation and human health. Clin Dermatol. 1998;16:83-9

14. Fitzpatrick TB. The validity and practicality of sun-reactive skin types I through VI. Arch Dermatol. 1988;124:869-71.

15. Serup J, Agner T. Colorimetric quantification of erythema--a comparison of two colorimeters (Lange Micro Color and Minolta Chroma Meter CR-
200) with a clinical scoring scheme and laser-Doppler flowmetry. Clin Exp Dermatol. 1990;15:267-72.

16. Del Bino S, Sok J, Bessac E, et al. Relationship between skin response to ultraviolet exposure and skin color type. Pigment Cell Res. 2006; 19:606-14.

17. Castanedo-Cázares JP, Torres-Alvarez B, Medellín-Pérez ME, et al. Conocimientos y actitudes de la población mexicana con respecto a la radiación solar. Gac Med Mex. 2006:142:451-5.

18. Jablonski NG, Chaplin G. Human skin pigmentation as an adaptation to UV radiation. Proc Natl Acad Sci U S A. 2010;107 Suppl:8962-8.

19. Yun IS, Lee WJ, Rah DK, et al. Skin color analysis using a spectrophotometer in Asians. Skin Res Technol. 2010;16:311-5.

20. Castanedo-Cázares JP, Torres-Álvarez B, Portales-González B, et al. Análisis de la radiación solar ultravioleta acumulada en México. Rev Med Inst Mex Seguro Soc. 2016;54:26-31.

21. Costin GE, Hearing VJ. Human skin pigmentation: melanocytes modulate skin color in response to stress. FASEB J. 2007;21:976-94.

22. Tsuchida $Y$. The effect of aging and arteriosclerosis on human skin blood flow. J Dermatol Sci. 1993;5:175-81. 